

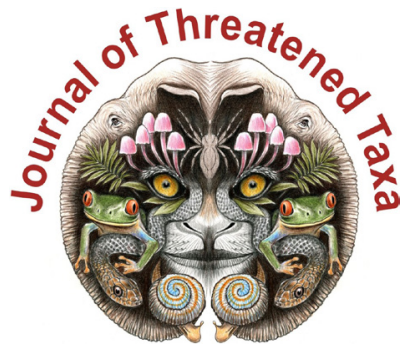

ISSN 0974-7907 (Online); ISSN $0974-7893$ (Print)

Publisher

Host

Wildlife Information Liaison Development Society

www.wild.zooreach.org

Zoo Outreach Organization www.zooreach.org

No. 12, Thiruvannamalai Nagar, Saravanampatti - Kalapatti Road, Saravanampatti, Coimbatore, Tamil Nadu 641035, India

Ph: +91 9385339863 | www.threatenedtaxa.org

Email: sanjay@threatenedtaxa.org

EDITORS

\section{Founder \& Chief Editor}

Dr. Sanjay Molur

Wildlife Information Liaison Development (WILD) Society \& Zoo Outreach Organization (ZOO),

12 Thiruvannamalai Nagar, Saravanampatti, Coimbatore, Tamil Nadu 641035, India

\section{Deputy Chief Editor}

Dr. Neelesh Dahanukar

Noida, Uttar Pradesh, India

\section{Managing Editor}

Mr. B. Ravichandran, WILD/ZOO, Coimbatore, India

\section{Associate Editors}

Dr. Mandar Paingankar, Government Science College Gadchiroli, Maharashtra 442605, India

Dr. Ulrike Streicher, Wildlife Veterinarian, Eugene, Oregon, USA

Ms. Priyanka Iyer, ZOO/WILD, Coimbatore, Tamil Nadu 641035, India

Dr. B.A. Daniel, ZOO/WILD, Coimbatore, Tamil Nadu 641035, India

\section{Editorial Board}

Dr. Russel Mittermeier

Executive Vice Chair, Conservation International, Arlington, Virginia 22202, USA

\section{Prof. Mewa Singh Ph.D., FASc, FNA, FNASc, FNAPsy}

Ramanna Fellow and Life-Long Distinguished Professor, Biopsychology Laboratory, and Institute of Excellence, University of Mysore, Mysuru, Karnataka 570006, India; Honorary Professor, Jawaharlal Nehru Centre for Advanced Scientific Research, Bangalore; and Adjunct Professor, National Institute of Advanced Studies, Bangalore

\section{Stephen D. Nash}

Scientific Illustrator, Conservation International, Dept. of Anatomical Sciences, Health Sciences Center, T-8, Room 045, Stony Brook University, Stony Brook, NY 11794-8081, USA

\section{Dr. Fred Pluthero}

Toronto, Canada

\section{Dr. Priya Davidar}

Sigur Nature Trust, Chadapatti, Mavinhalla PO, Nilgiris, Tamil Nadu 643223, India

\section{Dr. Martin Fisher}

Senior Associate Professor, Battcock Centre for Experimental Astrophysics, Cavendish

Laboratory, JJ Thomson Avenue, Cambridge CB3 OHE, UK

\section{Dr. John Fellowes}

Honorary Assistant Professor, The Kadoorie Institute, 8/F, T.T. Tsui Building, The University of Hong Kong, Pokfulam Road, Hong Kong

\section{Prof. Dr. Mirco Solé}

Universidade Estadual de Santa Cruz, Departamento de Ciências Biológicas, Vice-coordenado do Programa de Pós-Graduação em Zoologia, Rodovia Ilhéus/Itabuna, Km 16 (45662-000)

Salobrinho, Ilhéus - Bahia - Brasil

\section{Dr. Rajeev Raghavan}

Professor of Taxonomy, Kerala University of Fisheries \& Ocean Studies, Kochi, Kerala, India

\section{English Editors}

Mrs. Mira Bhojwani, Pune, India

Dr. Fred Pluthero, Toronto, Canad

Mr. P. Ilangovan, Chennai, India

Web Development

Mrs. Latha G. Ravikumar, ZOO/WILD, Coimbatore, India

\section{Typesetting}

Mr. Arul Jagadish, ZOO, Coimbatore, India

Mrs. Radhika, ZOO, Coimbatore, India

Mrs. Geetha, ZOO, Coimbatore India
Fundraising/Communications

Mrs. Payal B. Molur, Coimbatore, India

Subject Editors 2018-2020

Fungi

Dr. B. Shivaraju, Bengaluru, Karnataka, India

Dr. R.K. Verma, Tropical Forest Research Institute, Jabalpur, India

Dr. Vatsavaya S. Raju, Kakatiay University, Warangal, Andhra Pradesh, India

Dr. M. Krishnappa, Jnana Sahyadri, Kuvempu University, Shimoga, Karnataka, India

Dr. K.R. Sridhar, Mangalore University, Mangalagangotri, Mangalore, Karnataka, India

Dr. Gunjan Biswas, Vidyasagar University, Midnapore, West Bengal, India

\section{Plants}

Dr. G.P. Sinha, Botanical Survey of India, Allahabad, India

Dr. N.P. Balakrishnan, Ret. Joint Director, BSI, Coimbatore, India

Dr. Shonil Bhagwat, Open University and University of Oxford, UK

Prof. D.J. Bhat, Retd. Professor, Goa University, Goa, India

Dr. Ferdinando Boero, Università del Salento, Lecce, Italy

Dr. Dale R. Calder, Royal Ontaro Museum, Toronto, Ontario, Canada

Dr. Cleofas Cervancia, Univ. of Philippines Los Baños College Laguna, Philippines

Dr. F.B. Vincent Florens, University of Mauritius, Mauritius

Dr. Merlin Franco, Curtin University, Malaysia

Dr. V. Irudayaraj, St. Xavier's College, Palayamkottai, Tamil Nadu, India

Dr. B.S. Kholia, Botanical Survey of India, Gangtok, Sikkim, India

Dr. Pankaj Kumar, Kadoorie Farm and Botanic Garden Corporation, Hong Kong S.A.R., China

Dr. V. Sampath Kumar, Botanical Survey of India, Howrah, West Bengal, India

Dr. A.J. Solomon Raju, Andhra University, Visakhapatnam, India

Dr. Vijayasankar Raman, University of Mississippi, USA

Dr. B. Ravi Prasad Rao, Sri Krishnadevaraya University, Anantpur, India

Dr. K. Ravikumar, FRLHT, Bengaluru, Karnataka, India

Dr. Aparna Watve, Pune, Maharashtra, India

Dr. Qiang Liu, Xishuangbanna Tropical Botanical Garden, Yunnan, China

Dr. Noor Azhar Mohamed Shazili, Universiti Malaysia Terengganu, Kuala Terengganu, Malaysia

Dr. M.K. Vasudeva Rao, Shiv Ranjani Housing Society, Pune, Maharashtra, India

Prof. A.J. Solomon Raju, Andhra University, Visakhapatnam, India

Dr. Mandar Datar, Agharkar Research Institute, Pune, Maharashtra, India

Dr. M.K. Janarthanam, Goa University, Goa, India

Dr. K. Karthigeyan, Botanical Survey of India, India

Dr. Errol Vela, University of Montpellier, Montpellier, France

Dr. P. Lakshminarasimhan, Botanical Survey of India, Howrah, India

Dr. Larry R. Noblick, Montgomery Botanical Center, Miami, USA

Dr. K. Haridasan, Pallavur, Palakkad District, Kerala, India

Dr. Analinda Manila-Fajard, University of the Philippines Los Banos, Laguna, Philippines

Dr. P.A. Sinu, Central University of Kerala, Kasaragod, Kerala, India

Dr. Afroz Alam, Banasthali Vidyapith (accredited A grade by NAAC), Rajasthan, India

Dr. K.P. Rajesh, Zamorin's Guruvayurappan College, GA College PO, Kozhikode, Kerala, India

Dr. David E. Boufford, Harvard University Herbaria, Cambridge, MA 02138-2020, USA

Dr. Ritesh Kumar Choudhary, Agharkar Research Institute, Pune, Maharashtra, India

Dr. Navendu Page, Wildlife Institute of India, Chandrabani, Dehradun, Uttarakhand, India

\section{Invertebrates}

Dr. R.K. Avasthi, Rohtak University, Haryana, India

Dr. D.B. Bastawade, Maharashtra, India

Dr. Partha Pratim Bhattacharjee, Tripura University, Suryamaninagar, India

Dr. Kailash Chandra, Zoological Survey of India, Jabalpur, Madhya Pradesh, India

Dr. Ansie Dippenaar-Schoeman, University of Pretoria, Queenswood, South Africa

Dr. Rory Dow, National Museum of natural History Naturalis, The Netherlands

Dr. Brian Fisher, California Academy of Sciences, USA

Dr. Richard Gallon, llandudno, North Wales, LL30 1UP

Dr. Hemant V. Ghate, Modern College, Pune, India

Dr. M. Monwar Hossain, Jahangirnagar University, Dhaka, Bangladesh

Mr. Jatishwor Singh Irungbam, Biology Centre CAS, Branišovská, Czech Republic.

Dr. Ian J. Kitching, Natural History Museum, Cromwell Road, UK

Dr. George Mathew, Kerala Forest Research Institute, Peechi, India

For Focus, Scope, Aims, and Policies, visit https://threatenedtaxa.org/index.php/JoTT/aims_scope
For Article Submission Guidelines, visit https://threatenedtaxa.org/index.php/JoTT/about/submissions
For Policies against Scientific Misconduct, visit https://threatenedtaxa.org/index.php/JoTT/policies_various

continued on the back inside cover 


\title{
Butterflies of Amrabad Tiger Reserve, Telangana, India
}

\author{
Deepa Jaiswal $^{1}$ (D), B. Bharath ${ }^{2}$ (D) M. Karuthapandi ${ }^{3}$ (D), Shrikant Jadhav ${ }^{4}$ (D), S. Prabakaran ${ }^{5}$ (D)
}

\& S. Rehanuma Sulthana ${ }^{6}$ (D)

\footnotetext{
${ }^{1-6}$ Freshwater Biology Regional Centre, Zoological Survey of India, Hyderguda, Attapur, Hyderabad, Telangana 500048, India. ${ }^{1}$ deepajzsi@gmail.com, ${ }^{2}$ bhupathibharath23@gmail.com, ${ }^{3}$ kpandi83@gmail.com (corresponding author), ${ }^{4}$ shrikantjadhavzsi@gmail.com, ${ }^{5}$ prabakaranzsi@yahoo.co.in, ${ }^{6}$ rehansiddhu@gmail.com
}

\begin{abstract}
The butterfly diversity of Amrabad Tiger Reserve was assessed from March 2018 to February 2021. A total of 106 species belonging to the families Nymphalidae (36 species), Lycaenidae (30 species), Pieridae (18 species), Hesperiidae (14 species), and Papilionidae ( 8 species) were recorded. Of these, 12 species belonged to the Schedules I, II \& IV of the Indian Wildlife Protection Act, and four were common in this region.
\end{abstract}

Keywords: Butterfly diversity, Hesperiidae, Lycaenidae, Nymphalidae, Papilionidae, Pieridae, status.

Telangana state having a wide range of ecosystems is rich in its biodiversity (Khartade et al. 2019). Approximately, $26,900 \mathrm{~km}^{2}$ area constituting $24 \%$ of the total area are under forest. There are 12 protected areas, which consists of seven wildlife sanctuaries, three national parks and two tiger reserves. Amrabad Tiger Reserve is located in the northern part of Nallamala Hills, Eastern Ghats and on the banks of river Krishna. It has an area of $2,611.39 \mathrm{~km}^{2}$ with deciduous forest harbouring a wide variety of flora and fauna. It was previously a part of Nagarjunasagar Srisailam Tiger Reserve (NSTR), Andhra Pradesh. After bifurcation of Andhra Pradesh, the area of NSTR falling under the newly formed Telangana state was designated as Amrabad Tiger Reserve.
A total of 2,450 faunal species have been reported so far from the state of Telangana (Chandra et al. 2021), including 165 species of butterflies (Sailu et al. 2021). Earlier studies from adjacent habitats of the Eastern Ghats by Rao et al. (2004) reported 89 butterfly species from NSTR, Andhra Pradesh; Raju et al. (2003) reported 68 species from Visakhapatnam; Venkataramana (2010) reported 70 species from Visakhapatnam, Ananthagiri and Ratnagiri hills of Eastern Ghats; Ramamurty et al. (2013) reported 78 species from Visakhapatnam, Andhra Pradesh; and Goswami et al. (2018) recorded 102 species of butterflies from northern Eastern Ghats of Andhra Pradesh. In this study, an attempt has been made to document the butterfly diversity of Amrabad Tiger Reserve, Telangana.

\section{MATERIALS AND METHOdS}

Sampling of butterflies was made in 20 different sampling stations on a seasonal basis to cover different stretches of the tiger reserve. Observations were made by transact method from $0700-0900 \mathrm{~h}$ at least to cover 5 $\mathrm{km}$ of every sampling station, and randomised sampling from 9-11am and 4-6pm during the years 2018-2021 (Table 1, Figure 1). Butterflies were observed using

Citation: Jaiswal, D., B. Bharath, M. Karuthapandi, S. Jadhav, S. Prabakaran \& S.R. Sulthana (2021). Butterflies of Amrabad Tiger Reserve, Telangana, India. Journal of Threatened Taxa 13(13): 20090-20097. https://doi.org/10.11609/jott.6434.13.13.20090-20097

Copyright: @ Jaiswal et al. 2021. Creative Commons Attribution 4.0 International License. JoTT allows unrestricted use, reproduction, and distribution of this article in any medium by providing adequate credit to the author(s) and the source of publication.

Funding: None.

Competing interests: The authors declare no competing interests.

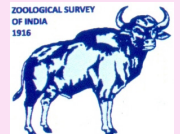

Acknowledgements: The authors are very grateful to the Director, Zoological Survey of India, Kolkata for the constant encouragement and support. We are thankful to the PCCF(H), Forest Department, Telangana, Conservator of forest, District forest officer, field director and range officers of Amrabad Tiger Reserve for their extended support and necessary permission for access of the reserve. We are also thank Mr. C. Shiva Shankar for his support during field survey. 


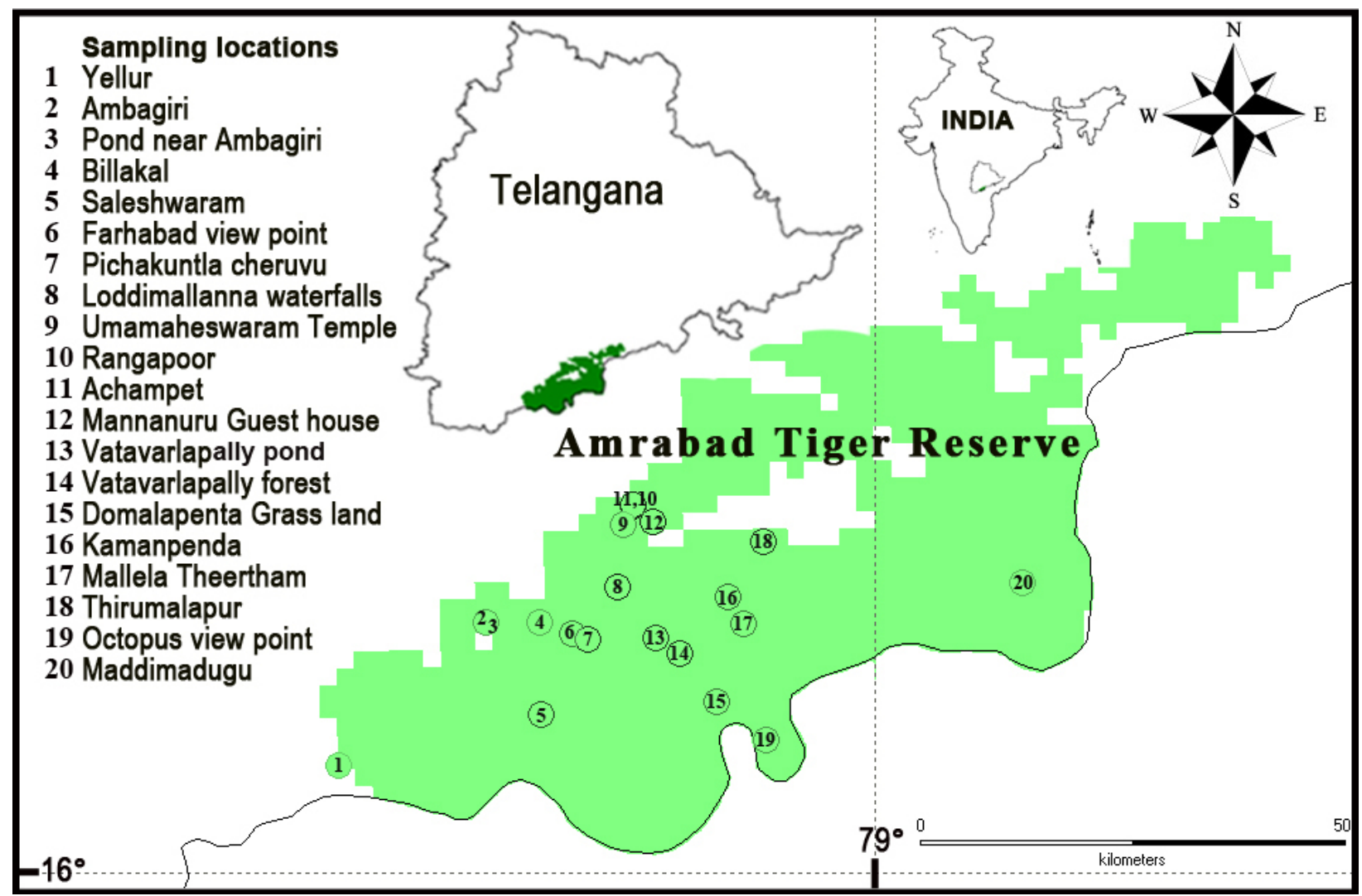

Figure 1. Map showing the sampling location of the Amrabad Tiger Reserve, Telangana.

Nikon binoculars and photographed by Nikon D500 DSLR camera. Collections were made with the help of a sweep net, samples were dry preserved and deposited in the museum collections of Zoological Survey of India, Freshwater Biology Regional Centre, Hyderabad. Species classification and nomenclature were after Kunte (2000), Kehimkar (2008), and Bhakare \& Ogale (2018).

\section{RESULTS AND DISCUSSION}

A total of 106 species of butterflies belonging to 68 genera, five families (Table 2, Image 1-106) were recorded from the Amrabad Tiger Reserve. Of these, specimens of 85 species have been collected and preserved. The remaining species were only photographed. Among the various families recorded, Nymphalidae with 36 species and 22 genera, showed high diversity followed by Lycaenidae (30 species) (Figure 2). Among these, 12 species belonged to the schedules I, II \& IV of the Indian Wildlife Protection Act (1972). Four species namely, viz., Dark Glass Blue Zizeeria karsandra, Peacock Pansy Junonia almana, Yellow Pansy Junonia hierta, and Small Grass Yellow Eurema brigitta were common, and assessed as 'Least Concern' as per IUCN Red List.

The maximum number of species was observed from

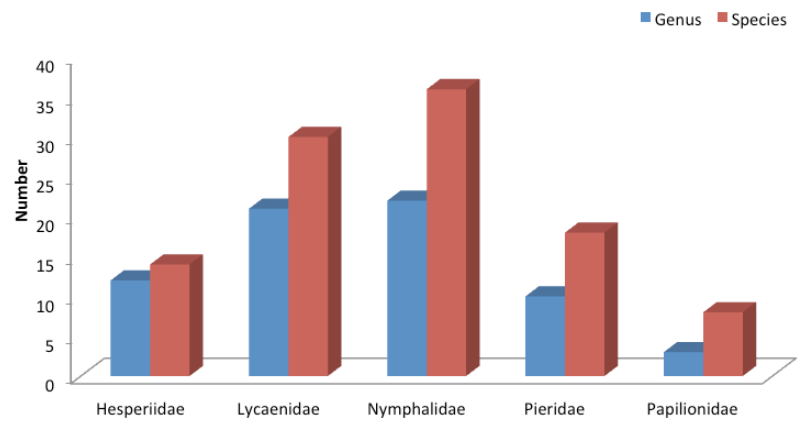

Figure 2. Family-wise species composition of butterflies of Amrabad Tiger Reserve.

Umamaheswaram temple with 35 species, followed by Saleshwaram with 25 species, Pichakuntla cheruvu and Billakal with 21 species each. Most of the species are common, and seven were observed rarely, such as Caprona ransonnettii from Mallelatheertham waterfalls, Neptis jumbah from Domalapenta grassland, Lethe europa, Phaedym columella, Spindasis schistacea from Saleshwaram, Polyura agrarian from Umamaheswaram, Colotis fausta from Pichakuntla cheruvu, Appias libythea from Ambagiri and Kamanpenda. 
Table 1. Sampling locations of Amrabad Tiger Reserve, Telangana.

\begin{tabular}{|c|c|c|c|}
\hline & Locality & Longitude (E) & Latitude (N) \\
\hline 1 & Achampet & 78.7377 & 16.3930 \\
\hline 2 & Ambagiri & 78.5792 & 16.2678 \\
\hline 3 & Thirumalapur & 78.8775 & 16.3554 \\
\hline 4 & Billakal & 78.6369 & 16.2678 \\
\hline 5 & Domalapenta Grass land & 78.8264 & 16.1831 \\
\hline 6 & Farhabad view point & 78.6714 & 16.2561 \\
\hline 7 & Kamanpenda & 78.8391 & 16.2958 \\
\hline 8 & Loddimallanna waterfalls & 78.7203 & 16.3061 \\
\hline 9 & Maddimadugu & 79.1551 & 16.3115 \\
\hline 10 & Mallela Theertham Waterfalls & 78.8558 & 16.2672 \\
\hline 11 & Mannanuru Guest house & 78.7583 & 16.3758 \\
\hline 12 & Octopus view point & 78.8800 & 16.1415 \\
\hline 13 & Pichakuntla cheruvu & 78.6889 & 16.2506 \\
\hline 14 & Pond near Ambagiri & 78.5792 & 16.2675 \\
\hline 15 & Rangapoor & 78.7375 & 16.3939 \\
\hline 16 & Saleshwaram & 78.6392 & 16.1689 \\
\hline 17 & Umamaheswaram Temple & 78.7267 & 16.3728 \\
\hline 18 & Vatavarlapally forest & 78.7881 & 16.2350 \\
\hline 19 & Vatavarlapally pond & 78.7614 & 16.2514 \\
\hline 20 & Yellur & 78.4217 & 16.1153 \\
\hline
\end{tabular}

The 165 species of butterflies have been recorded from Telangana, and the species recorded in this study represent $64 \%$ of those reported from the state. Eightynine species were reported from NSTR and of these 20 were not observed in the present study. The present study documented 34 new additions to the list of butterfly species of Amrabad Tiger Reserve. Butterflies recorded in this study showed $69 \%$ similarity with species recorded in Rao et al. (2004), 50\% with Goswami et al. (2018), 68\% with Raju et al. (2003), and 62\% with Venkataramana (2010). It shows that the northern and southern Eastern Ghats species distribution are approximately $45 \%$ dissimilar, it could be due to variation in habitat, host plant and climatic conditions.

The family Nymphalidae are dominant in the tropical region because most of them are polyphagous in nature and thus able to survive in all habitats. Additionally, many species of this family are strong, active fliers able to search for resources in large areas (Easwaran \& Pramod 2005; Krishnakumar et al. 2008). The high proportion of nymphalidae observed might also be due to the availability of a variety of host plants in Amrabad Tiger Reserve. The predominance of Nymphalidae over other butterfly groups in the Western Ghats has earlier been
Table 2 List of Butterfly species recorded from Amrabad Tiger Reserve, Telangana

\begin{tabular}{|c|c|c|c|c|}
\hline & Scientific name & Common name & $\begin{array}{l}\text { IUCN } \\
\text { Status }\end{array}$ & $\begin{array}{l}\text { WPA } \\
\text { Status }\end{array}$ \\
\hline & Hesperiidae & & & \\
\hline 1 & $\begin{array}{l}\text { Borbo cinnara } \\
\text { (Wallace, 1866) }\end{array}$ & Rice Swift & & \\
\hline 2 & $\begin{array}{l}\text { Caltoris kumara } \\
\text { (Moore, 1878) }\end{array}$ & Blank Swift & & \\
\hline 3 & $\begin{array}{l}\text { Caprona agama } \\
\text { (Moore, 1858) }\end{array}$ & Spotted Angle & & \\
\hline 4 & $\begin{array}{l}\text { Caprona ransonnettii } \\
\text { (R.Felder, 1868) }\end{array}$ & Golden Angle & & \\
\hline 5 & $\begin{array}{l}\text { Hasora chromus } \\
\text { (Cramer, 1780) }\end{array}$ & $\begin{array}{l}\text { Common } \\
\text { Banded Awl }\end{array}$ & & \\
\hline 6 & $\begin{array}{l}\text { Matapa aria (Moore, } \\
1866 \text { ) }\end{array}$ & $\begin{array}{l}\text { Common Red } \\
\text { Eye }\end{array}$ & & \\
\hline 7 & $\begin{array}{l}\text { Lambrix salsala } \\
\text { (Moore, 1866) }\end{array}$ & Chestnut Bob & & \\
\hline 8 & $\begin{array}{l}\text { Parnara ganga } \\
\text { Evans, } 1937\end{array}$ & $\begin{array}{l}\text { Continental } \\
\text { Swift }\end{array}$ & & \\
\hline 9 & $\begin{array}{l}\text { Pelopidas mathias } \\
\text { (Fabricius, 1798) }\end{array}$ & $\begin{array}{l}\text { Small-Branded } \\
\text { Swift }\end{array}$ & & \\
\hline 10 & $\begin{array}{l}\text { Pelopidas } \\
\text { subochracea (Moore, } \\
1878 \text { ) }\end{array}$ & $\begin{array}{l}\text { Large Banded } \\
\text { Swift }\end{array}$ & & \\
\hline 11 & $\begin{array}{l}\text { Spialia galba } \\
\text { (Fabricius, 1793) }\end{array}$ & Indian Skipper & & \\
\hline 12 & $\begin{array}{l}\text { Suastus gremius } \\
\text { (Fabricius, 1798) }\end{array}$ & Indian Palm Bob & & \\
\hline 13 & $\begin{array}{l}\text { Telicota bambusae } \\
\text { (Moore, 1878) }\end{array}$ & Dark Palm Dart & & \\
\hline \multirow[t]{2}{*}{14} & $\begin{array}{l}\text { Udaspes folus } \\
\text { (Cramer, 1775) }\end{array}$ & Grass Demon & & \\
\hline & Lycaenidae & & & \\
\hline 15 & $\begin{array}{l}\text { Azanus jesous } \\
\text { (Guérin-Méneville, } \\
1849 \text { ) }\end{array}$ & $\begin{array}{l}\text { African Babul } \\
\text { Blue }\end{array}$ & & \\
\hline 16 & $\begin{array}{l}\text { Azanus ubaldus } \\
\text { (Stoll, 1782) }\end{array}$ & $\begin{array}{l}\text { Bright Babul } \\
\text { Blue }\end{array}$ & & \\
\hline 17 & $\begin{array}{l}\text { Azanus uranus Butler, } \\
1886\end{array}$ & Dull Babul Blue & & \\
\hline 18 & $\begin{array}{l}\text { Caleta decidia } \\
\text { (Hewitson, 1876) } \\
\end{array}$ & Angled Pierrot & & \\
\hline 19 & $\begin{array}{l}\text { Castalius rosimon } \\
\text { (Fabricius, 1775) }\end{array}$ & $\begin{array}{l}\text { Common } \\
\text { Pierrot }\end{array}$ & & $\begin{array}{l}\text { Schedule I } \\
\text { Species }\end{array}$ \\
\hline 20 & $\begin{array}{l}\text { Catochrysops } \\
\text { panormus (C.Felder, } \\
\text { 1860) }\end{array}$ & $\begin{array}{l}\text { Silver Forget- } \\
\text { Me-Not }\end{array}$ & & \\
\hline 21 & $\begin{array}{l}\text { Catochrysops strabo } \\
\text { (Fabricius, 1793) }\end{array}$ & Forget-Me-Not & & \\
\hline 22 & $\begin{array}{l}\text { Chilades lajus (Stoll, } \\
1780 \text { ) }\end{array}$ & $\begin{array}{l}\text { Indian Lime } \\
\text { blue }\end{array}$ & & \\
\hline 23 & $\begin{array}{l}\text { Chilades pandava } \\
\text { (Horsfield, 1829) }\end{array}$ & Plains Cupid & & \\
\hline 24 & $\begin{array}{l}\text { Chilades parrhasius } \\
\text { (Fabricius, 1793) }\end{array}$ & Small Cupid & & \\
\hline 25 & $\begin{array}{l}\text { Curetis thetis (Drury, } \\
1773 \text { ) }\end{array}$ & Indian Sunbeam & & \\
\hline 26 & $\begin{array}{l}\text { Euchrysops cnejus } \\
\text { (Fabricius, 1798) }\end{array}$ & Gram Blue & & $\begin{array}{l}\text { Schedule II } \\
\text { Species }\end{array}$ \\
\hline 27 & $\begin{array}{l}\text { Everes lacturuns } \\
\text { (Godart, 1824) }\end{array}$ & Indian Cupid & & \\
\hline 28 & $\begin{array}{l}\text { Freyeria putli (Kollar, } \\
\text { 1844) }\end{array}$ & $\begin{array}{l}\text { Small Grass } \\
\text { Jewel }\end{array}$ & & \\
\hline
\end{tabular}




\begin{tabular}{|c|c|c|c|c|}
\hline & Scientific name & Common name & $\begin{array}{l}\text { IUCN } \\
\text { Status }\end{array}$ & $\begin{array}{l}\text { WPA } \\
\text { Status }\end{array}$ \\
\hline 29 & $\begin{array}{l}\text { Jamides bochus } \\
\text { (Stoll, 1782) }\end{array}$ & Dark Cerulean & & \\
\hline 30 & $\begin{array}{l}\text { Jamides celeno } \\
\text { (Cramer 1775) }\end{array}$ & $\begin{array}{l}\text { Common } \\
\text { Cerulean }\end{array}$ & & \\
\hline 31 & $\begin{array}{l}\text { Lampides boeticus } \\
\text { (Linnaeus, 1767) }\end{array}$ & Pea Blue & & $\begin{array}{c}\text { Schedule II } \\
\text { Species }\end{array}$ \\
\hline 32 & $\begin{array}{l}\text { Leptotes plinius } \\
\text { (Fabricius, 1793) }\end{array}$ & Zebra Blue & & \\
\hline 33 & $\begin{array}{l}\text { Prosotas dubiosa } \\
\text { indica (Evans, 1925) }\end{array}$ & $\begin{array}{l}\text { Tailless Line } \\
\text { Blue }\end{array}$ & & \\
\hline 34 & $\begin{array}{l}\text { Prosotas nora } \\
\text { (C.Felder, 1860) }\end{array}$ & $\begin{array}{l}\text { Common Line } \\
\text { Blue }\end{array}$ & & \\
\hline 35 & $\begin{array}{l}\text { Rathinda amor } \\
\text { (Fabricius, 1775) }\end{array}$ & Monkey Puzzle & & \\
\hline 36 & $\begin{array}{l}\text { Spindasis ictis } \\
\text { (Hewitson, 1865) }\end{array}$ & $\begin{array}{l}\text { Common Shot } \\
\text { Silverline }\end{array}$ & & \\
\hline 37 & $\begin{array}{l}\text { Spindasis schistacea } \\
\text { (Moore, 1881) }\end{array}$ & $\begin{array}{l}\text { Plumbeous } \\
\text { Silverline }\end{array}$ & & \\
\hline 38 & $\begin{array}{l}\text { Spindasis vulcanus } \\
\text { (Fabricius, 1775) }\end{array}$ & $\begin{array}{l}\text { Common } \\
\text { Silverline }\end{array}$ & & \\
\hline 39 & $\begin{array}{l}\text { Talicada nyseus } \\
\text { (Guérin-Méneville, } \\
\text { 1843) }\end{array}$ & Red Pierrot & & \\
\hline 40 & $\begin{array}{l}\text { Tarucus nara (Kollar, } \\
\text { 1848) }\end{array}$ & Striped Pierrot & & \\
\hline 41 & $\begin{array}{l}\text { Virachola isocrates } \\
\text { (Fabricius, 1793) }\end{array}$ & $\begin{array}{l}\text { Common Guava } \\
\text { blue }\end{array}$ & & \\
\hline 42 & $\begin{array}{l}\text { Zizeeria karsandra } \\
\text { (Moore, 1865) }\end{array}$ & Dark Grass Blue & $\begin{array}{c}\text { Least } \\
\text { Concern }\end{array}$ & \\
\hline 43 & $\begin{array}{l}\text { Zizina otis (Fabricius, } \\
\text { 1787) }\end{array}$ & $\begin{array}{l}\text { Lesser Grass } \\
\text { Blue }\end{array}$ & & \\
\hline \multirow[t]{2}{*}{44} & $\begin{array}{l}\text { Zizula hylax } \\
\text { (Fabricius, 1775) }\end{array}$ & Tiny Grass Blue & & \\
\hline & Nymphalidae & & & \\
\hline 45 & $\begin{array}{l}\text { Acraca terpsicore } \\
\text { (Linnaeus, 1758) }\end{array}$ & Tawny Coster & & \\
\hline 46 & $\begin{array}{l}\text { Ariadne merione } \\
\text { (Cramer, 1777) }\end{array}$ & Common Castor & & \\
\hline 47 & $\begin{array}{l}\text { Ariadne ariadne } \\
\text { (Linnaeus, 1763) }\end{array}$ & Angled Castor & & \\
\hline 48 & $\begin{array}{l}\text { Byblia ilithyia (Drury, } \\
1773 \text { ) }\end{array}$ & Joker & & \\
\hline 49 & $\begin{array}{l}\text { Charaxes solon } \\
\text { (Fabricius, 1793) }\end{array}$ & Black Rajah & & $\begin{array}{c}\text { Schedule II } \\
\text { Species }\end{array}$ \\
\hline 50 & $\begin{array}{l}\text { Danaus chrysippus } \\
\text { (Linnaeus, 1758) }\end{array}$ & Plain Tiger & & \\
\hline 51 & $\begin{array}{l}\text { Danaus genutia } \\
\text { (Cramer, 1779) }\end{array}$ & Striped Tiger & & \\
\hline 52 & $\begin{array}{l}\text { Euploea core } \\
\text { (Cramer, 1780) }\end{array}$ & Common Crow & & \\
\hline 53 & $\begin{array}{l}\text { Euploea sylvester } \\
\text { (Fabricius, 1793) }\end{array}$ & $\begin{array}{l}\text { Double-Branded } \\
\text { Crow }\end{array}$ & & \\
\hline 54 & $\begin{array}{l}\text { Euthalia aconthea } \\
\text { (Cramer, 1777) }\end{array}$ & Common Baron & & $\begin{array}{c}\text { Schedule II } \\
\text { Species }\end{array}$ \\
\hline 55 & $\begin{array}{l}\text { Hypolimnas bolina } \\
\text { (Linnaeus, 1758) }\end{array}$ & Great Eggfly & & \\
\hline 56 & $\begin{array}{l}\text { Hypolimnas misippus } \\
\text { (Linnaeus, 1764) }\end{array}$ & Danaid Eggfly & & \begin{tabular}{|c|} 
Schedule I \\
Species
\end{tabular} \\
\hline 57 & $\begin{array}{l}\text { Junonia almana } \\
\text { (Linnaeus, 1758) }\end{array}$ & Peacock Pansy & $\begin{array}{l}\text { Least } \\
\text { Concern }\end{array}$ & \\
\hline 58 & $\begin{array}{l}\text { Junonia atlites } \\
\text { (Linnaeus, 1763) }\end{array}$ & Gray Pansy & & \\
\hline 59 & $\begin{array}{l}\text { Junonia hierta } \\
\text { (Fabricius, 1798) }\end{array}$ & Yellow Pansy & $\begin{array}{c}\text { Least } \\
\text { Concern }\end{array}$ & \\
\hline 60 & $\begin{array}{l}\text { Junonia iphita } \\
\text { (Cramer, 1779) }\end{array}$ & Chocolate pansy & & \\
\hline
\end{tabular}

\begin{tabular}{|c|c|c|c|c|}
\hline & Scientific name & Common name & $\begin{array}{l}\text { IUCN } \\
\text { Status }\end{array}$ & $\begin{array}{l}\text { WPA } \\
\text { Status }\end{array}$ \\
\hline 61 & $\begin{array}{l}\text { Junonia lemonias } \\
\text { (Linnaeus, 1758) }\end{array}$ & Lemon Pansy & & \\
\hline 62 & $\begin{array}{l}\text { Junonia orithya } \\
\text { (Linnaeus, 1758) }\end{array}$ & Blue Pansy & & \\
\hline 63 & $\begin{array}{l}\text { Lethe europa } \\
\text { (Fabricius, 1775) }\end{array}$ & $\begin{array}{l}\text { Bamboo Tree } \\
\text { Brown }\end{array}$ & & \\
\hline 65 & $\begin{array}{l}\text { Melanitis leda } \\
\text { (Linnaeus, 1758) }\end{array}$ & $\begin{array}{l}\text { Common } \\
\text { Evening Brown }\end{array}$ & & \\
\hline 66 & $\begin{array}{l}\text { Moduza procris } \\
\text { (Cramer, 1777) }\end{array}$ & Commander & & \\
\hline 67 & $\begin{array}{l}\text { Mycalesis mineus } \\
\text { (Linnaeus, 1758) }\end{array}$ & $\begin{array}{l}\text { Dark Banded } \\
\text { Bush Brown }\end{array}$ & & $\begin{array}{l}\text { Schedule II } \\
\text { Species }\end{array}$ \\
\hline 67 & $\begin{array}{l}\text { Mycalesis perseus } \\
\text { (Fabricius, 1775) }\end{array}$ & $\begin{array}{l}\text { Common } \\
\text { Bushbrown }\end{array}$ & & \\
\hline 68 & $\begin{array}{l}\text { Neptis hylas } \\
\text { (Linnaeus, 1758) }\end{array}$ & Common Sailer & & \\
\hline 69 & $\begin{array}{l}\text { Neptis jumbah } \\
\text { Moore, } 1858\end{array}$ & $\begin{array}{l}\text { Chestnut } \\
\text { Streaked Sailer }\end{array}$ & & $\begin{array}{l}\text { Schedule I } \\
\text { Species }\end{array}$ \\
\hline 70 & $\begin{array}{l}\text { Phaedyma columella } \\
\text { (Cramer, 1780) }\end{array}$ & $\begin{array}{l}\text { Short Banded } \\
\text { Sailer }\end{array}$ & & \\
\hline 71 & $\begin{array}{l}\text { Parantica aglea } \\
\text { (Stoll, 1782) }\end{array}$ & Glassy Tiger & & \\
\hline 72 & $\begin{array}{l}\text { Phalantha phalantha } \\
\text { (Drury, 1773) }\end{array}$ & $\begin{array}{l}\text { Common } \\
\text { Leopard }\end{array}$ & & \\
\hline 73 & $\begin{array}{l}\text { Polyura agraria } \\
\text { (Swinhoe, 1887) }\end{array}$ & $\begin{array}{l}\text { Anomalous } \\
\text { Nawab }\end{array}$ & & \\
\hline 74 & $\begin{array}{l}\text { Polyura athamas } \\
\text { (Drury, 1773) }\end{array}$ & $\begin{array}{l}\text { Common } \\
\text { Nawab }\end{array}$ & & $\begin{array}{l}\text { Schedule II } \\
\text { Species }\end{array}$ \\
\hline 75 & $\begin{array}{l}\text { Symphaedra nais } \\
\text { (Forster, 1771) }\end{array}$ & Baronet & & \\
\hline 76 & $\begin{array}{l}\text { Tirumala limniace } \\
\text { (Cramer, 1775) }\end{array}$ & Blue Tiger & & \\
\hline 77 & $\begin{array}{l}\text { Tirumala } \\
\text { septentrionis (Butler, } \\
1874 \text { ) }\end{array}$ & Dark Blue tiger & & \\
\hline 78 & $\begin{array}{l}\text { Vanessa cardui } \\
\text { (Linnaeus, 1758) }\end{array}$ & Painted Lady & & \\
\hline 79 & $\begin{array}{l}\text { Ypthima asterope } \\
\text { (Klug, 1832) }\end{array}$ & $\begin{array}{l}\text { Common Three- } \\
\text { Ring }\end{array}$ & & \\
\hline \multirow[t]{2}{*}{80} & $\begin{array}{l}\text { Ypthima baldus } \\
\text { (Fabricius,1775) }\end{array}$ & $\begin{array}{l}\text { Common Five } \\
\text { Ring }\end{array}$ & & \\
\hline & Pieridae & & & \\
\hline 81 & $\begin{array}{l}\text { Appias albina } \\
\text { (Boisoduval, 1836) }\end{array}$ & $\begin{array}{l}\text { Common } \\
\text { Albatross }\end{array}$ & & \\
\hline 82 & $\begin{array}{l}\text { Appias libythea } \\
\text { (Fabricius, 1775) }\end{array}$ & Striped Albatros & & $\begin{array}{l}\text { Schedule } \\
\text { IV Species }\end{array}$ \\
\hline 83 & $\begin{array}{l}\text { Belenois aurota } \\
\text { (Fabricius, 1793) }\end{array}$ & Pioneer & & \\
\hline 84 & $\begin{array}{l}\text { Catopsilia pyranthe } \\
\text { (Linnaeus, 1758) }\end{array}$ & $\begin{array}{l}\text { Mottled } \\
\text { Emigrant }\end{array}$ & & \\
\hline 85 & $\begin{array}{l}\text { Catospilia pomona } \\
\text { (Fabricius, 1775) }\end{array}$ & $\begin{array}{l}\text { Common } \\
\text { Emigrant }\end{array}$ & & \\
\hline 86 & $\begin{array}{l}\text { Cepora nerissa } \\
\text { (Fabricius, 1775) }\end{array}$ & Common Gull & & $\begin{array}{l}\text { Schedule II } \\
\text { Species }\end{array}$ \\
\hline 87 & $\begin{array}{l}\text { Colotis aurora } \\
\text { (Cramer, 1780) }\end{array}$ & Plain Orange Tip & & \\
\hline 88 & $\begin{array}{l}\text { Colotis danae } \\
\text { (Fabricius, 1775) }\end{array}$ & Crimson Tip & & \\
\hline 89 & $\begin{array}{l}\text { Colotis etrida } \\
\text { (Boisoduval, 1836) }\end{array}$ & Little Orange Tip & & \\
\hline 90 & $\begin{array}{l}\text { Colotis fausta } \\
\text { (Olivier, 1804) }\end{array}$ & $\begin{array}{l}\text { Large Salmon } \\
\text { Arab }\end{array}$ & & \\
\hline 91 & $\begin{array}{l}\text { Delias eucharis } \\
\text { (Drury, 1773) }\end{array}$ & $\begin{array}{l}\text { Common } \\
\text { Jezebel }\end{array}$ & & \\
\hline 92 & $\begin{array}{l}\text { Eurema brigitta } \\
\text { (Stoll, 1780) }\end{array}$ & $\begin{array}{l}\text { Small Grass } \\
\text { Yellow }\end{array}$ & $\begin{array}{l}\text { Least } \\
\text { Concern }\end{array}$ & \\
\hline
\end{tabular}




\begin{tabular}{|c|c|c|c|c|}
\hline & Scientific name & Common name & $\begin{array}{l}\text { IUCN } \\
\text { Status }\end{array}$ & $\begin{array}{l}\text { WPA } \\
\text { Status }\end{array}$ \\
\hline 93 & $\begin{array}{l}\text { Eurema hecabe } \\
\text { (Linnaeus, 1758) }\end{array}$ & $\begin{array}{l}\text { Common Grass } \\
\text { Yellow }\end{array}$ & & \\
\hline 94 & $\begin{array}{l}\text { Eurema laeta } \\
\text { (Boisduval, 1836) }\end{array}$ & $\begin{array}{l}\text { Spotless Grass } \\
\text { Yellow }\end{array}$ & & \\
\hline 95 & $\begin{array}{l}\text { Leptosia nina } \\
\text { (Fabricius, 1793) }\end{array}$ & Psyche & & \\
\hline 96 & $\begin{array}{l}\text { Ixias marianne } \\
\text { (Cramer, 1779) }\end{array}$ & $\begin{array}{l}\text { White Orange } \\
\text { Tip }\end{array}$ & & \\
\hline 97 & $\begin{array}{l}\text { Ixias pyrene } \\
\text { (Linnaeus, 1764) }\end{array}$ & $\begin{array}{l}\text { Yellow Orange } \\
\text { Tip }\end{array}$ & & \\
\hline \multirow[t]{2}{*}{98} & $\begin{array}{l}\text { Pareronia hippia } \\
\text { (Fabricius, 1787) }\end{array}$ & $\begin{array}{l}\text { Common } \\
\text { Wanderer }\end{array}$ & & \\
\hline & Papilionidae & & & \\
\hline 99 & $\begin{array}{l}\text { Graphium } \\
\text { agamemnon } \\
\text { (Linnaeus, 1758) }\end{array}$ & Tailed Jay & & \\
\hline 100 & $\begin{array}{l}\text { Graphium doson } \\
\text { (C\&R Felder, 1864) }\end{array}$ & Common Jay & & \\
\hline 101 & $\begin{array}{l}\text { Graphium nomius } \\
\text { (Esper, 1799) }\end{array}$ & Spot Swordtail & & \\
\hline 102 & $\begin{array}{l}\text { Pachliopta } \\
\text { aristolochiae } \\
\text { (Fabricius, 1775) }\end{array}$ & Common Rose & & \\
\hline 103 & $\begin{array}{l}\text { Pachliopta hector } \\
\text { (Linnaeus, 1758) }\end{array}$ & Crimson Rose & & $\begin{array}{l}\text { Schedule I } \\
\text { Species }\end{array}$ \\
\hline 104 & $\begin{array}{l}\text { Papilio demoleus } \\
\text { Linnaeus, } 1758\end{array}$ & Lime Butterfly & & \\
\hline 105 & $\begin{array}{l}\text { Papilio polytes } \\
\text { Linnaeus, } 1758\end{array}$ & $\begin{array}{l}\text { Common } \\
\text { Mormon }\end{array}$ & & \\
\hline 106 & $\begin{array}{l}\text { Papilio crino } \\
\text { Fabricius, } 1793\end{array}$ & $\begin{array}{l}\text { Common } \\
\text { Banded Peacock }\end{array}$ & & \\
\hline
\end{tabular}

WPA-Wildlife Protection Act | IUCN-International Union for Conservation of Nature.

reported by (Kunte 1997; Easwaran \& Pramod 2005). Amrabad Tiger Reserve has mixed vegetation supporting rich species diversity. The increase in butterfly diversity may be due to favourable climatic conditions, availability of more number of host plants and vegetation cover of herbs, shrubs and trees for nectaring of butterflies (Tiple 2009). Since, the Amrabad Tiger Reserve hosts the scheduled and least concern species, conservation measures to ensure habitat protection in the tiger reserve are essential. However, further studies on ecology, threats and conservation of butterfly needs to be focused.

\section{REFERENCES}

Bhakare, M \& H. Ogale (2018). A Guide to the Butterflies of Western Ghats (India) includes Butterfiles of Kerala, Tamilnadu, Karnataka, Goa, Maharashtra and Gujarat state. Published by Milind Bhakare \& Hemant Olgale, Maharashtra, 496pp.

Chandra, K., J. Deepa, C. Raghunathan, S.S. Jadhav \& M. Karuthapandi (2021). Current status of faunal diversity in Telangana. Zoological Survey of India, Kolkata, 394pp.

Easwaran, R. \& P. Pramod (2005). Structure of butter flies community of Anaikatty hills, Western Ghats, Zoo's Print Journal 20: 1939-1942. https://doi.org/10.11609/JoTT.ZPJ.1330.1939-42

Goswami, R., O. Thorat, V. Aditya \& S.N. Karimbumkara (2018). A preliminary checklist of butterflies from the northern Eastern Ghats with notes on new and significant species records including three new reports for peninsular India. Journal of Threatened Taxa 10(13): 12769-12791. https://doi.org/10.11609/jott.3730.10.13.127691279

Kehimkar, I. (2008). The Book of Indian Butterflies. Bombay Natural History Society, Oxford University press, Walton Street, Oxford, New York, 497pp.

Khartade, K.S., S. Reddy, G. Sailu, J. Swamy, L. Rasingam, C. Srinivasilu, J. Deepa, R. Deepak, T. Farida, M. Karuthapandi, S. Shilpi, S.S. Jadhav \& V.V. Rao (eds.) (2019). Telangana State Biodiversity Field Guide. Telangana State Biodiversity Board, Hyderabad, 293pp.

Krishnakumar, N., K. Kumaraguru, A. Thiyagesan \& V. Asokan (2008). Diversity of papilionid butterflies in the Indira Gandhi Wildlife Sanctuary, Western Ghats, Southern India. Tiger Paper 35: 1-8. https://doi.org/10.5281/zenodo.4322288

Kunte, K. (1997). Seasonal patterns in butterfly abundance and species diversity in four tropical habitats in northern Western Ghats. Journal of Bioscience 22: 593-603. https://doi.org/10.1007/BF02703397

Kunte, K. (2000). Butterflies of Peninsular India. Indian Academy of Sciences, Universities Press (India) Limited, Hyderabad, India, 254pp.

Raju, A.J.S., S.P. Rao \& V. Ezradanam (2003). Some ecological notes on the butterflies of Visakhapatnam, Andhra Pradesh. Zoos' Print Journal 18(6): 1126-1128. https://doi.org/10.11609/JoTT. ZPJ.18.6.1126-8

Ramamurty, M., A. Rohini, S.T.P.L. Ushasri, Ch. Girijarani, P. Sharon, S.Pavani \& U.J. Rani (2013). Preliminary study on Butterfly Diversity in the Biodiversity Park of Rani Chandramani Devi Government Hospital, Visakhapatnam, Andhra Pradesh. Advances in Pollen Spore Research XXXI: 151-159.

Rao, K.T., M.P. Raju, S.M.M. Javed \& I. Sivaramakrishna (2004). A checklist of Butterflies of Nagarjunasagar Srisaliam Tiger Reserve, Andhra Pradesh. Zoos' Print Journal 19(2): 1713-1715. https://doi. org/10.11609/JoTT.ZPJ.1184.1713-5

Sailu, G., B. Bharath \& M. Karuthapandi (2021). Insecta: Lepidoptera (Butterflies), pp. 251-261. In: Chandra, K., J. Deepa, C. Raghunathan, S.S. Jadhav \& M. Karuthapandi (eds.). Current status of Faunal diversity in Telangana. Zoological Survey of India, Kolkata.

Tiple, A.D. (2009). Butterflies from Nagpur city, Central India: Diversity, population, nectar and larval host plants and the implications for conservation. PhD Thesis. RTM Nagpur University, Nagpur, India, $146 p p$.

Venkataramana, S.P. (2010). Biodiversity and Conservation of Butterflies in the Eastern Ghats. The Ecoscan 4(1): 59-67. 

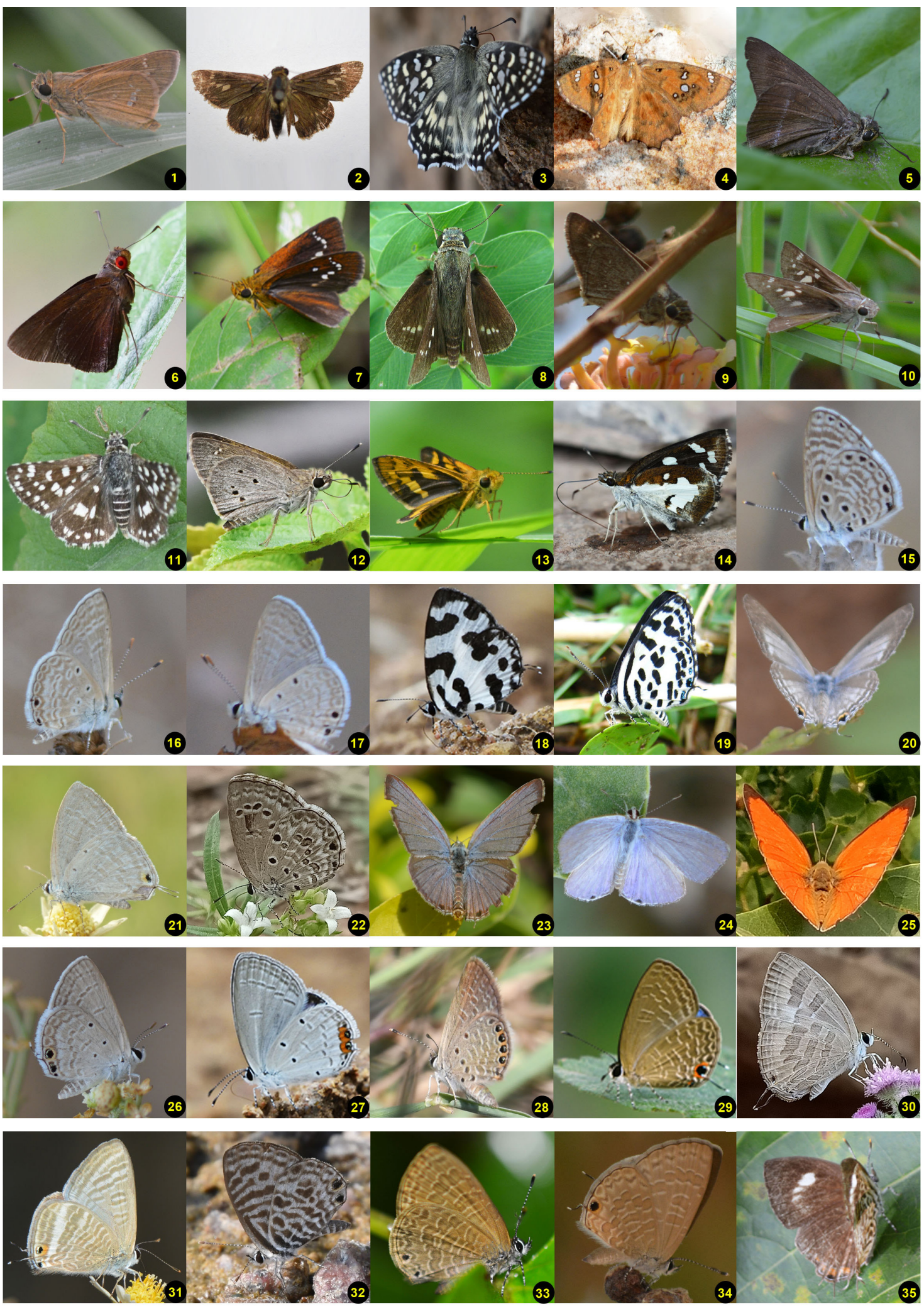

Image 1-35. 1-Borbo cinnara | 2-Caltoris kumara | 3-Caprona agama | 4-Caprona ransonnettii | 5-Hasora chromus | 6-Matapa aria | 7-Lambrix salsala | 8-Parnara ganga | 9-Pelopidas mathias | 10-Pelopidas subochracea | 11-Spialia galba | 12-Suastus gremius | 13Telicota bambusae | 14-Udaspes folus | 15-Azanus jesous | 16-Azanus ubaldus | 17-Azanus uranus | 18-Calet decidia | 19-Castalius rosimon | 20-Catochrysops panormus | 21-Catochrysops strabo | 22-Chilades lajus | 23-Chilades pandava | 24-Chilades parrhasius | 25-Curetis thetis | 26-Euchrysops cnejus | 27-Everes lacturuns | 28-Freyeria putli | 29-Jamides bochus | 30-Jamides celeno | 31Lampides boeticus | 32-Leptotes plinius | 33-Prosotas dubiosa indica | 34-Prosotas nora | 35-Rathinda amor. (C) Authors 

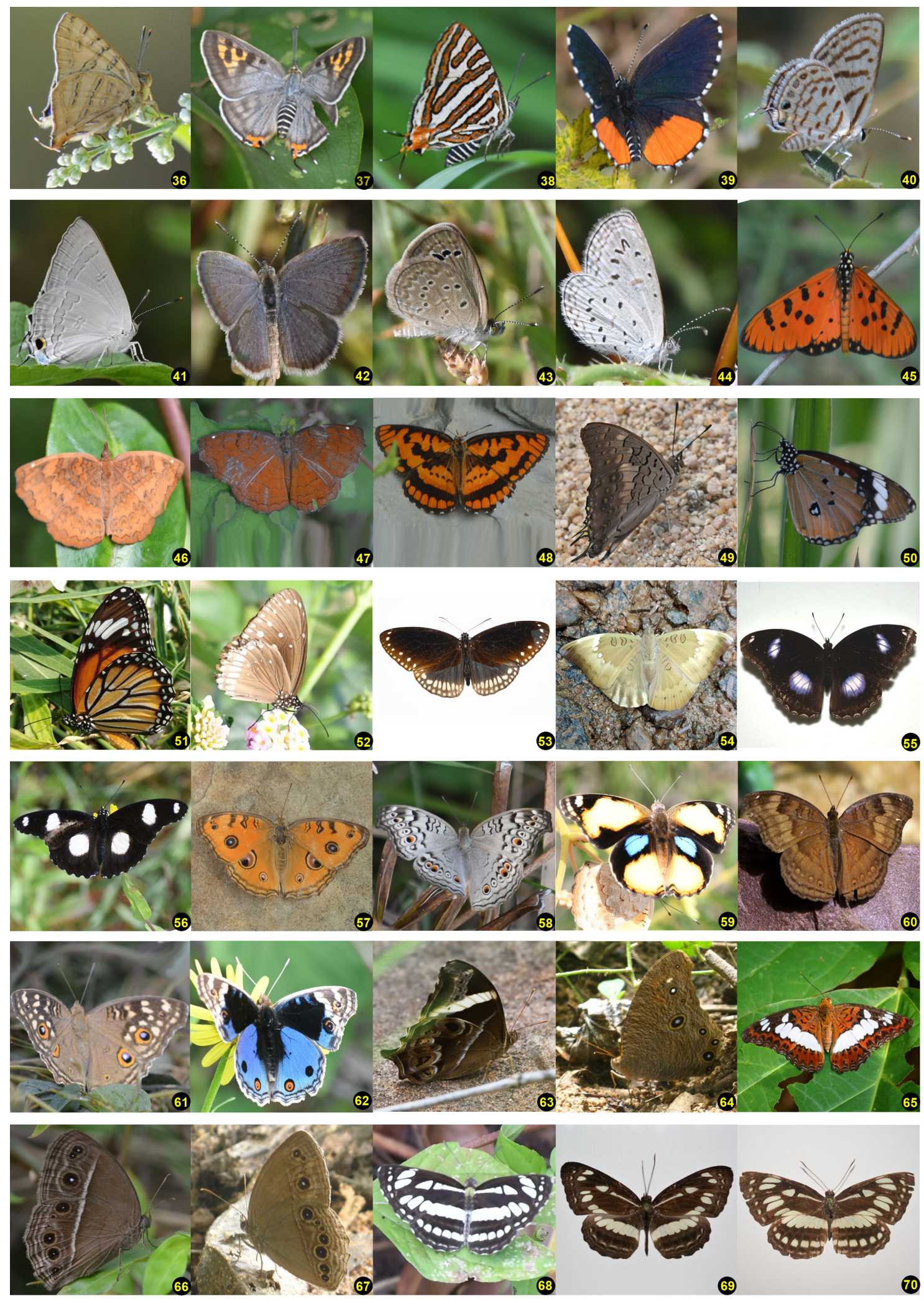

Image 36-70. 36-Spindasis ictis | 37-Spindasis schistacea | 38-Spindasis vulcanus | 39-Talicada nyseus | 40-Tarucus nara | 41Virachola isocrates | 42-Zizeeria karsandra | 43-Zizina otis | 44-Zizula hylax | 45-Acraca terpsicore | 46-Ariadne merione | 47-Ariadne ariadne | 48-Byblia ilithyia | 49-Charaxes solon | 50-Danaus chrysippus | 51-Danaus genutia | 52-Euploea core | 53-Euploea sylvester | 54-Euthalia aconthea | 55-Hypolimnas bolina | 56-Hypolimnas misippus | 57-Junonia almana | 58-Junonia atlites | 59-Junonia hierta | 60-Junonia iphita | 61-Junonia lemonias | 62-Junonia orithya |63-Lethe europa |64-Melanitis leda | 65-Moduza procris | 66-Mycalesis mineus | 67-Mycalesis perseus | 68-Neptis hylas | 69-Neptis jumbah | 70-Phaedyma columella. @ Authors 

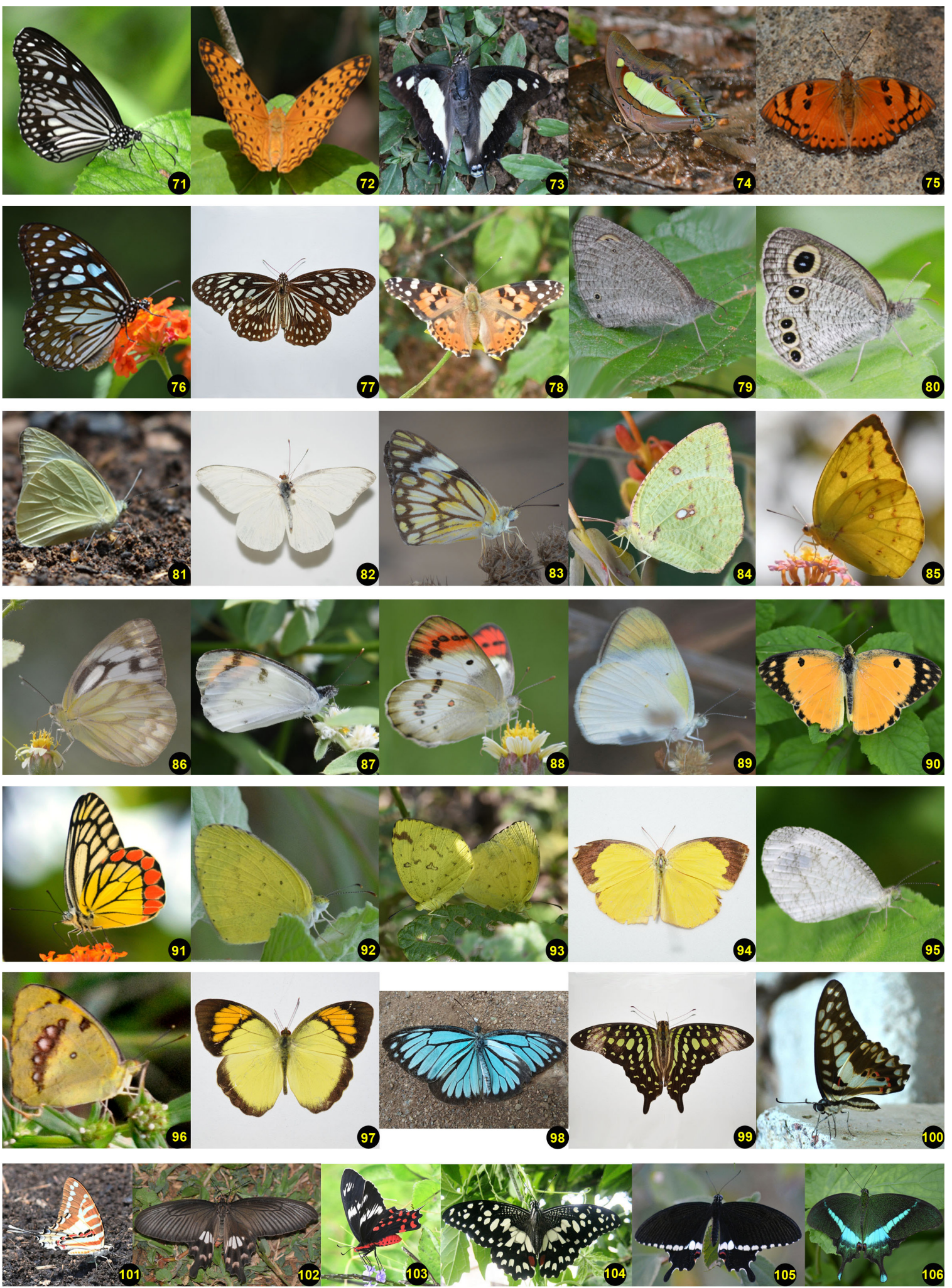

Image 71-106. 71-Parantica aglea | 72-Phalantha phalantha | 73-Polyura agraria | 74-Polyura athamas | 75-Symphaedra nais | 76Tirumala limniace | 77-Tirumala septentrionis | 78-Vanessa cardui | 79-Ypthima asterope | 80-Ypthima baldus | 81-Appias albina | 82-Appias libythea | 83-Belenois aurota | 84-Catopsilia pyranthe | 85-Catospilia Pomona | 86-Cepora nerissa | 87-Colotis aurora | 88-Colotis danae | 89-Colotis etrida | 90-Colotis fausta | 91-Delias eucharis | 92-Eurema brigitta | 93-Eurema hecabe | 94-Eurema laeta | 95-Leptosia nina | 96-Ixias marianne | 97-Ixias pyrene | 98-Pareronia hippia | 99-Graphium agamemnon | 100-Graphium doson | 101-Graphium nomius | 102-Pachliopta aristolochiae | 103-Pachliopta hector | 104-Papilio demoleus | 105-Papilio polytes | 106-Papilio crino. C Authors 
Dr. John Noyes, Natural History Museum, London, UK

Dr. Albert G. Orr, Griffith University, Nathan, Australia

Dr. Sameer Padhye, Katholieke Universiteit Leuven, Belgium

Dr. Nancy van der Poorten, Toronto, Canada

Dr. Kareen Schnabel, NIWA, Wellington, New Zealand

Dr. R.M. Sharma, (Retd.) Scientist, Zoological Survey of India, Pune, India

Dr. Manju Siliwal, WILD, Coimbatore, Tamil Nadu, India

Dr. G.P. Sinha, Botanical Survey of India, Allahabad, India

Dr. K.A. Subramanian, Zoological Survey of India, New Alipore, Kolkata, India

Dr. P.M. Sureshan, Zoological Survey of India, Kozhikode, Kerala, India

Dr. R. Varatharajan, Manipur University, Imphal, Manipur, India

Dr. Eduard Vives, Museu de Ciències Naturals de Barcelona, Terrassa, Spain

Dr. James Young, Hong Kong Lepidopterists' Society, Hong Kong

Dr. R. Sundararaj, Institute of Wood Science \& Technology, Bengaluru, India

Dr. M. Nithyanandan, Environmental Department, La Ala Al Kuwait Real Estate. Co. K.S.C.,

Kuwait

Dr. Himender Bharti, Punjabi University, Punjab, India

Mr. Purnendu Roy, London, UK

Dr. Saito Motoki, The Butterfly Society of Japan, Tokyo, Japan

Dr. Sanjay Sondhi, TITLI TRUST, Kalpavriksh, Dehradun, India

Dr. Nguyen Thi Phuong Lien, Vietnam Academy of Science and Technology, Hanoi, Vietnam

Dr. Nitin Kulkarni, Tropical Research Institute, Jabalpur, India

Dr. Robin Wen Jiang Ngiam, National Parks Board, Singapore

Dr. Lional Monod, Natural History Museum of Geneva, Genève, Switzerland.

Dr. Asheesh Shivam, Nehru Gram Bharti University, Allahabad, India

Dr. Rosana Moreira da Rocha, Universidade Federal do Paraná, Curitiba, Brasi

Dr. Kurt R. Arnold, North Dakota State University, Saxony, Germany

Dr. James M. Carpenter, American Museum of Natural History, New York, USA

Dr. David M. Claborn, Missouri State University, Springfield, USA

Dr. Kareen Schnabel, Marine Biologist, Wellington, New Zealand

Dr. Amazonas Chagas Júnior, Universidade Federal de Mato Grosso, Cuiabá, Brasil

Mr. Monsoon Jyoti Gogoi, Assam University, Silchar, Assam, India

Dr. Heo Chong Chin, Universiti Teknologi MARA (UiTM), Selangor, Malaysia

Dr. R.J. Shiel, University of Adelaide, SA 5005, Australia

Dr. Siddharth Kulkarni, The George Washington University, Washington, USA

Dr. Priyadarsanan Dharma Rajan, ATREE, Bengaluru, India

Dr. Phil Alderslade, CSIRO Marine And Atmospheric Research, Hobart, Australia

Dr. John E.N. Veron, Coral Reef Research, Townsville, Australia

Dr. Daniel Whitmore, State Museum of Natural History Stuttgart, Rosenstein, Germany.

Dr. Yu-Feng Hsu, National Taiwan Normal University, Taipei City, Taiwan

Dr. Keith V. Wolfe, Antioch, California, USA

Dr. Siddharth Kulkarni, The Hormiga Lab, The George Washington University, Washington,

D.C., USA

Dr. Tomas Ditrich, Faculty of Education, University of South Bohemia in Ceske

Budejovice, Czech Republic

Dr. Mihaly Foldvari, Natural History Museum, University of Oslo, Norway

Dr. V.P. Uniyal, Wildlife Institute of India, Dehradun, Uttarakhand 248001, India

Dr. John T.D. Caleb, Zoological Survey of India, Kolkata, West Bengal, India

Dr. Priyadarsanan Dharma Rajan, Ashoka Trust for Research in Ecology and the Environment

(ATREE), Royal Enclave, Bangalore, Karnataka, India

\section{Fishes}

Dr. Neelesh Dahanukar, IISER, Pune, Maharashtra, India

Dr. Topiltzin Contreras MacBeath, Universidad Autónoma del estado de Morelos, México

Dr. Heok Hee Ng, National University of Singapore, Science Drive, Singapore

Dr. Rajeev Raghavan, St. Albert's College, Kochi, Kerala, India

Dr. Robert D. Sluka, Chiltern Gateway Project, A Rocha UK, Southall, Middlesex, UK

Dr. E. Vivekanandan, Central Marine Fisheries Research Institute, Chennai, India

Dr. Davor Zanella, University of Zagreb, Zagreb, Croatia

Dr. A. Biju Kumar, University of Kerala, Thiruvananthapuram, Kerala, India

Dr. Akhilesh K.V., ICAR-Central Marine Fisheries Research Institute, Mumbai Research

Centre, Mumbai, Maharashtra, India

Dr. J.A. Johnson, Wildlife Institute of India, Dehradun, Uttarakhand, India

Amphibians

Dr. Sushil K. Dutta, Indian Institute of Science, Bengaluru, Karnataka, India

Dr. Annemarie Ohler, Muséum national d'Histoire naturelle, Paris, France

\section{Reptiles}

Dr. Gernot Vogel, Heidelberg, Germany

Dr. Raju Vyas, Vadodara, Gujarat, India

Dr. Pritpal S. Soorae, Environment Agency, Abu Dubai, UAE.

Prof. Dr. Wayne J. Fuller, Near East University, Mersin, Turkey

Prof. Chandrashekher U. Rivonker, Goa University, Taleigao Plateau, Goa. India

Dr. S.R. Ganesh, Chennai Snake Park, Chennai, Tamil Nadu, India

Dr. Himansu Sekhar Das, Terrestrial \& Marine Biodiversity, Abu Dhabi, UAE
Birds

Dr. Hem Sagar Baral, Charles Sturt University, NSW Australia

Dr. Chris Bowden, Royal Society for the Protection of Birds, Sandy, UK

Dr. Priya Davidar, Pondicherry University, Kalapet, Puducherry, India

Dr. J.W. Duckworth, IUCN SSC, Bath, UK

Dr. Rajah Jayapal, SACON, Coimbatore, Tamil Nadu, India

Dr. Rajiv S. Kalsi, M.L.N. College, Yamuna Nagar, Haryana, India

Dr. V. Santharam, Rishi Valley Education Centre, Chittoor Dt., Andhra Pradesh, India

Dr. S. Balachandran, Bombay Natural History Society, Mumbai, India

Mr. J. Praveen, Bengaluru, India

Dr. C. Srinivasulu, Osmania University, Hyderabad, India

Dr. K.S. Gopi Sundar, International Crane Foundation, Baraboo, USA

Dr. Gombobaatar Sundev, Professor of Ornithology, Ulaanbaatar, Mongolia

Prof. Reuven Yosef, International Birding \& Research Centre, Eilat, Israel

Dr. Taej Mundkur, Wetlands International, Wageningen, The Netherlands

Dr. Carol Inskipp, Bishop Auckland Co., Durham, UK

Dr. Tim Inskipp, Bishop Auckland Co, Durham, UK

Dr. V. Gokula, National College, Tiruchirappalli, Tamil Nadu, India

Dr. Arkady Lelej, Russian Academy of Sciences, Vladivostok, Russia

Dr. Simon Dowell, Science Director, Chester Zoo, UK

Dr. Mário Gabriel Santiago dos Santos, Universidade de Trás-os-Montes e Alto Douro,

Quinta de Prados, Vila Real, Portugal

Dr. Grant Connette, Smithsonian Institution, Royal, VA, USA

Dr. M. Zafar-ul Islam, Prince Saud Al Faisal Wildlife Research Center, Taif, Saudi Arabia

Mammals

Dr. Giovanni Amori, CNR - Institute of Ecosystem Studies, Rome, Italy

Dr. Anwaruddin Chowdhury, Guwahati, India

Dr. David Mallon, Zoological Society of London, UK

Dr. Shomita Mukherjee, SACON, Coimbatore, Tamil Nadu, India

Dr. Angie Appel, Wild Cat Network, Germany

Dr. P.O. Nameer, Kerala Agricultural University, Thrissur, Kerala, India

Dr. Ian Redmond, UNEP Convention on Migratory Species, Lansdown, UK

Dr. Heidi S. Riddle, Riddle's Elephant and Wildlife Sanctuary, Arkansas, USA

Dr. Karin Schwartz, George Mason University, Fairfax, Virginia.

Dr. Lala A.K. Singh, Bhubaneswar, Orissa, India

Dr. Mewa Singh, Mysore University, Mysore, India

Dr. Paul Racey, University of Exeter, Devon, UK

Dr. Honnavalli N. Kumara, SACON, Anaikatty P.O., Coimbatore, Tamil Nadu, India

Dr. Nishith Dharaiya, HNG University, Patan, Gujarat, India

Dr. Spartaco Gippoliti, Socio Onorario Società Italiana per la Storia della Fauna "Giuseppe

Altobello", Rome, Italy

Dr. Justus Joshua, Green Future Foundation, Tiruchirapalli, Tamil Nadu, India

Dr. H. Raghuram, The American College, Madurai, Tamil Nadu, India

Dr. Paul Bates, Harison Institute, Kent, UK

Dr. Jim Sanderson, Small Wild Cat Conservation Foundation, Hartford, USA

Dr. Dan Challender, University of Kent, Canterbury, UK

Dr. David Mallon, Manchester Metropolitan University, Derbyshire, UK

Dr. Brian L. Cypher, California State University-Stanislaus, Bakersfield, CA

Dr. S.S. Talmale, Zoological Survey of India, Pune, Maharashtra, India

Prof. Karan Bahadur Shah, Budhanilakantha Municipality, Kathmandu, Nepal

Dr. Susan Cheyne, Borneo Nature Foundation International, Palangkaraja, Indonesia

Dr. Hemanta Kafley, Wildlife Sciences, Tarleton State University, Texas, USA

\section{Other Disciplines}

Dr. Aniruddha Belsare, Columbia MO 65203, USA (Veterinary)

Dr. Mandar S. Paingankar, University of Pune, Pune, Maharashtra, India (Molecular)

Dr. Jack Tordoff, Critical Ecosystem Partnership Fund, Arlington, USA (Communities)

Dr. Ulrike Streicher, University of Oregon, Eugene, USA (Veterinary)

Dr. Hari Balasubramanian, EcoAdvisors, Nova Scotia, Canada (Communities)

Dr. Rayanna Hellem Santos Bezerra, Universidade Federal de Sergipe, São Cristóvão, Brazil

Dr. Jamie R. Wood, Landcare Research, Canterbury, New Zealand

Dr. Wendy Collinson-Jonker, Endangered Wildlife Trust, Gauteng, South Africa

Dr. Rajeshkumar G. Jani, Anand Agricultural University, Anand, Gujarat, India

Dr. O.N. Tiwari, Senior Scientist, ICAR-Indian Agricultural Research Institute (IARI), New

Delhi, India

Dr. L.D. Singla, Guru Angad Dev Veterinary and Animal Sciences University, Ludhiana, India

Dr. Rupika S. Rajakaruna, University of Peradeniya, Peradeniya, Sri Lanka

Dr. Bahar Baviskar, Wild-CER, Nagpur, Maharashtra 440013, India

Reviewers 2018-2020

Due to pausity of space, the list of reviewers for $2018-2020$ is available online.

The opinions expressed by the authors do not reflect the views of the Journal of Threatened Taxa, Wildlife Information Liaison Development Society, Zoo Outreach Organization, or any of the partners. The journal, the publisher, the host, and the partners are not responsible for the accuracy of the political boundaries shown in the maps by the authors.

Journal of Threatened Taxa is indexed/abstracted in Bibliography of Systematic Mycology, Biological Abstracts, BIOSIS Previews, CAB Abstracts, EBSCO, Google Scholar, Index Copernicus, Index Fungorum, JournalSeek, National Academy of Agricultural Sciences, NewJour, OCLC WorldCat, SCOPUS, Stanford University Libraries, Virtual Library of Biology, Zoological Records.

NAAS rating (India) 5.64
Print copies of the Journal are available at cost. Write to:

The Managing Editor, JoTT,

c/o Wildlife Information Liaison Development Society,

No. 12, Thiruvannamalai Nagar, Saravanampatti - Kalapatti Road,

Saravanampatti, Coimbatore, Tamil Nadu 641035, India

ravi@threatenedtaxa.org 


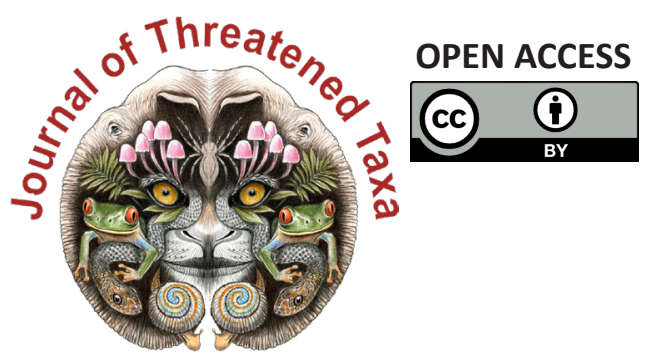

www.threatenedtaxa.org

The Journal of Threatened Taxa (JoTT) is dedicated to building evidence for conservation globally by publishing peer-reviewed articles online every month at a reasonably rapid rate at www.threatenedtaxa.org. All articles published in JoTT are registered under Creative Commons Attribution 4.0 International License unless otherwise mentioned. JoTT allows allows unrestricted use, reproduction, and distribution of articles in any medium by providing adequate credit to the author(s) and the source of publication.

ISSN 0974-7907 (Online) I ISSN $0974-7893$ (Print)

\section{November 2021 | Vol. 13 | No. 13 | Pages: 19887-20142 \\ Date of Publication: 26 November 2021 (Online \& Print) DOI: 10.11609/jott.2021.13.13.19887-20142}

\section{Article}

An inventory of geometrid moths (Lepidoptera: Geometroidea: Geometridae) of KalakadMundanthurai Tiger Reserve, India

- Geetha Iyer, Dieter Stüning \& Sanjay Sondhi, Pp. 19887-19920

\section{Communications}

Roadkills of Lowland Tapir Tapirus terrestris (Mammalia: Perissodactyla: Tapiridae) in one of its last refuges in the Atlantic Forest

- Aureo Banhos, Andressa Gatti, Marcelo Renan de Deus Santos, Leonardo Merçon,

Ilka Westermeyer, Natália Carneiro Ardente, Luis Francisco Oliveira Pereira Gonzaga, Lucas Mendes Barreto, Lucas Damásio, Tomas Lima Rocha, Vitor Roberto Schettino, Renata Valls, Helena Godoy Bergallo, Marcos Vinicius Freitas Silva, Athelson Stefanon Bittencourt, Danielle de Oliveira Moreira \& Ana Carolina Srbek-Araujo, Pp. 19921-19929

Scientific contributions and learning experiences of citizen volunteers with a small cat project in Sanjay Gandhi National Park, Mumbai, India

- Shomita Mukherjee, R. Nandini, P.V. Karunakaran \& Nayan Khanolkar, Pp. 19930-19936

Seasonal food preferences and group activity pattern of Blackbuck Antilope cervicapra (L., 1758) (Mammalia: Cetartiodactyla: Bovidae) in a semi-arid region of western Haryana, India

- Vikram Delu, Dharambir Singh, Sumit Dookia, Priya \& Kiran, Pp. 19937-19947

Studies on the habitats of Grey Francolin Francolinus pondicerianus (J.F. Gmelin, 1789) (Galliformes: Phasianidae) in northern districts of Tamil Nadu, India

- M. Pandian, Pp. 19948-19955

Recovery of vulture population in roosting and scavenging areas of Bastar and Bijapur, Chhattisgarh, India

- Sushil Kumar Dutta, Muntaz Khan, P.R.S. Nagi, Santosh Durgam \& Surabhi Dutta, Pp. 19956-19963

A geographical assessment of Chariganga and Arpara Beel (wetlands) of Nadia, West Bengal as a habitat of wetland birds

- Mehedi Hasan Mandal, Arindam Roy \& Giyasuddin Siddique, Pp. 19964-19975

Phenotypic plasticity in Barilius vagra (Hamilton, 1822) (Teleostei: Danionidae) from two geographically distinct river basins of Indian Himalaya

- Sumit Kumar, Sharali Sharma \& Deepak Singh, Pp. 19976-19984

Taxonomic notes, a new species, and a key to Indian species of the click beetle genus Cryptalaus Ôhira, 1967 (Coleoptera: Elateridae: Agrypninae)

- Harshad Parekar \& Amol Patwardhan, Pp. 19985-19999

Niche overlap of benthic macrofauna in a tropical estuary: diurnal variation

- Mário Herculano de Oliveira, Lidiane Gomes de Lima, Caroline Stefani da Silva Lima, Jéssica de Oliveira Lima Gomes, Franciely Ferreira Paiva, Graciele de Barros, Carlinda Railly Medeiros \& Joseline Molozzi, Pp. 20000-20010

Diversity of aquatic insects and biomonitoring of water quality in the upper Ganga River, a Ramsar site: a preliminary assessment

- Kritish De, Arkojyoti Sarkar, Kritika Singh, Virendra Prasad Uniyal, Jeyaraj Antony Johnson \& Syed Ainul Hussain, Pp. 20011-20018

Patterns of forest cover loss in the terrestrial Key Biodiversity Areas in the Philippines: critical habitat conservation priorities

- Bernard Peter O. Daipan, Pp. 20019-20032

The woody flora of Shettihalli Wildlife Sanctuary, central Western Ghats of Karnataka, India - A checklist

- Kanda Naveen Babu, Kurian Ayushi, Vincy K. Wilson, Narayanan Ayyappan \&

Narayanaswamy Parthasarathy, Pp. 20033-20055

Reproductive biology of Ophiorrhiza caudata C.E.C.Fisch. (Rubiaceae), an endemic and endangered creeping perennial herb of the Western Ghats, India

- Maria Theresa, Appukuttan Kamalabai Sreekala \& Jayalakshmi Mohanlal, Pp. 20056-20065
Short Communications

Successful rescue, medical management, rehabilitation, and translocation of a Red Panda Ailurus fulgens (Mammalia: Carnivora: Ailuridae) in Arunachal Pradesh, India - Jahan Ahmed, Sorang Tadap, Millo Tasser, Koj Rinya, Nekibuddin Ahmed \& Sunil Kyarong, Pp. 20066-20071

A rare photographic record of Eurasian Otter Lutra lutra with a note on its habitat from the Bhagirathi Basin, western Himalaya, India

- Ranjana Pal, Aashna Sharma, Vineet Kumar Dubey, Tapajit Bhattacharya, Jeyaraj Antony Johnson, Kuppusamy Sivakumar \& Sambandam Sathyakumar, Pp. 20072-20077

The first record of Medog Gliding Frog Rhacophorus translineatus Wu, 1977 (Anura: Rhacophoridae) from Chhukha District, Bhutan

- Sonam Lhendup \& Bal Krishna Koirala, Pp. 20078-20083

First record of a freshwater crab, Maydelliathelphusa masoniana (Henderson, 1893) (Decapoda: Brachyura: Gecarcinucidae) from West Bengal, India

- Ram Krishna Das, Pp. 20084-20089

Butterflies of Amrabad Tiger Reserve, Telangana, India

- Deepa Jaiswal, B. Bharath, M. Karuthapandi, Shrikant Jadhav, S. Prabakaran \& S. Rehanuma Sulthana, Pp. 20090-20097

An enumeration of the flowering plants of Kyongnosla Alpine Sanctuary in eastern Sikkim, India

- Sudhansu Sekhar Dash, Subhajit Lahiri \& Ashiho Asoshii Mao, Pp. 20098-20117

A new record of psychrotrophic Paecilomyces formosus (Eurotiales: Ascomycota) from India: morphological and molecular characterization

- Skarma Nonzom \& Geeta Sumbali, Pp. 20118-20123

Notes

Study on incidence and pathology of gastrointestinal parasitic infections in Nilgai Boselaphus tragocamelus in Hisar, Haryana, India

- Maneesh Sharma, B.L. Jangir, D. Lather, G.A. Chandratre, V. Nehra, K.K. Jakhar \& G. Narang, Pp. 20124-20127

An unusual vocalization of Brown Hawk-Owl Ninox scutulata (Raffles, 1822) (Aves:

Strigiformes: Strigidae) recorded from Kerala, India

- Riju P. Nair \& Shine Raj Tholkudiyil, Pp. 20128-20129

New distribution data on the genus Maripanthus Maddison, 2020 (Araneae: Salticidae) from southern India

- A. Asima, John T.D. Caleb, Dhruv A. Prajapati \& G. Prasad, Pp. 20130-20132

On the IUCN status of Boesenbergia albolutea and B. rubrolutea (Zingiberaceae) and typification of $B$. rubrolutea

- K. Aishwarya \& M. Sabu, Pp. 20133-20135

New records of mass seeding Cephalostachyum latifolium Munro (Poaceae) along the midelevation broadleaved forest of Sarpang district, Bhutan

- Jigme Tenzin, Sangay Nidup \& Dago Dorji, Pp. 20136-20139

Response

If habitat heterogeneity is effective for conservation of butterflies in urban landscapes of Delhi, India?' Unethical publication based on data manipulation

- Sanjay Keshari Das \& Rita Singh, Pp. 20140-20142

Publisher \& Host
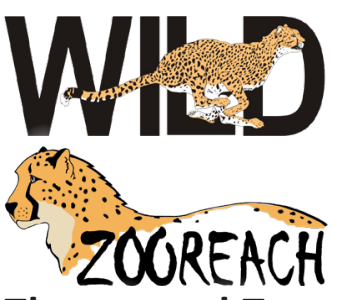

Threatened Taxa 